\title{
Low complexity MIMO detection using OSIC with conditional list generation
}

\author{
Wei Hou ${ }^{a)}$, Tadashi Fujino, and Toshiharu Kojima \\ Department of Communication Engineering and Informatics, \\ The University of Electro-Communications, \\ 1-5-1 Chofugaoka, Chofu, Tokyo 182-8585, Japan \\ a)houwei@uec.ac.jp
}

Abstract: This paper proposes a low complexity MIMO detection using ordering SIC (OSIC) with conditional list generation. Combined with lattice-reduction (LR) technique, OSIC further gains the BER performance compared to SIC, since the more reliable signal is prior to being detected. However OSIC based on LR (LR-OSIC) has inferior performance to the maximum likelihood (ML) detection in large size MIMO systems. In this paper, we investigate the mean square error in LR domain to evaluate the condition of the channel. The proposed detection applies the conditional list detection to update the estimate of the LR-OSIC. The simulation results exhibited that the proposed detection achieved near-ML performance in the $8 \times 8 \mathrm{MIMO}$ and required almost same complexity of the LR-OSIC in the high $E_{b} / N_{0}$ region.

Keywords: error propagation, list detection, MIMO, ML, OSIC

Classification: Wireless Communication Technologies

\section{References}

[1] X. Ma and W. Zhang, "Performance analysis for MIMO system with lattice-reduction aided linear equalization," IEEE Trans. Commun., vol. 56, pp. 309-318, Feb. 2008.

[2] K. Lee, J. Chun, and L. Hanzo, "Optimal lattice-reduction aided successive interference cancellation for MIMO Systems," IEEE Trans. Wireless Commun., vol. 6, no. 7, pp. 2438-2443, July 2007.

[3] H. Hassibi, "An efficient square-root algorithm for BLAST," Proc. IEEE Int. Conf. on Acoustic, Speech, Signal Processing, Istanbul, Turkey, pp. II737-II740, June 2000.

[4] T. Yamamoto and T. Fujino, "An improved lattice-reduction aided MMSE with successive interference cancellation," Proc. IEEE Int. Conf. on Advanced Technol. for Commun., Hanoi, Vietnam, pp. 45-49, Oct. 2008.

[5] G. H. Golub, C. F. Van Loan, Matrix Computations, The Johns Hopkins University Press, 3rd ed., Oct. 1996.

[6] D. Wübben, R. Böhnke, V. Kuhn, and K. D. Kammeyer, "MMSE-based lattice-reduction for near-ML detection of MIMO systems," Int. ITG Workshop on Smart Antennas, Munich, Germany, March 2004. 


\section{Introduction}

Recently, the use of lattice-reduction (LR) technique for date detection in the MIMO systems has been proposed in order to achieve the suboptimal BER performance with relatively low complexity [1]. Combining the LR algorithm with the linear detections such as zero-forcing (ZF) or minimum mean-square error (MMSE) and the non-linear detection like successive interference cancellation (SIC) can achieve the good BER performance, which are close to the ML detector in the small size MIMO system. In contrast, the BER improvements in the large size MIMO system are not sufficient [4]. The objective of the proposed detection is to provide the near-ML performance with lower complexity even in the large size MIMO system.

In this paper, we first apply the LR-OSIC, which is described in [2]. In the LR-OSIC, the estimated symbol is quantized using the rounding operation. Through the observation of the probability distribution of the difference between the estimated symbol and the rounding integer, we evaluate the channel condition. According to the mean square error of the signal vectors in the LR domain, we use the conditional list generation to update the estimate of the LR-OSIC. The simulation results verify that the proposed detection can achieve the near-ML performance in the $8 \times 8$ MIMO system and require almost same complexity of the LR-OSIC in the high $E_{b} / N_{0}$ region.

\section{System model and conventional detection}

\subsection{System model}

Consider a multiple antenna system with $M$ transmit and $N(N \geq M)$ receive antennas as

$$
\mathbf{y}^{c}=\mathbf{H}^{c} \mathbf{s}^{c}+\mathbf{z}^{c}
$$

where $\mathbf{H}^{c}=\left[\mathbf{h}_{1}^{c}, \ldots, \mathbf{h}_{M}^{c}\right]$ denotes the $N \times M$ channel matrix, of which the entries are of the i.i.d. complex Gaussian process with zero mean and unity variance. The receive signal vector is expressed as $\mathbf{y}^{c} \in \mathbb{C}^{N \times 1}$. The transmit signal vector is denoted as $\mathbf{s}^{c} \in \mathbb{S}^{M \times 1}$, where each symbol is chosen from a finite subset of the complex-valued integer set $\mathbb{S}$. The additive white Gaussian noise (AWGN) vector is represented as $\mathbf{z}^{c} \in \mathbb{C}^{N \times 1}$, of which each entry is assumed to be zero mean and variance of $\sigma^{2} / 2$ in each dimension.

As LR algorithm such as the famous LLL algorithm is generally modeled for real-valued system, Eq. (1) can be rewritten separating the real and imaginary components as

$$
\mathbf{y}=\mathbf{H s}+\mathbf{z}
$$

where the equivalent real-valued channel matrix and vectors are defined as

$$
\begin{aligned}
& \mathbf{H} \triangleq\left[\begin{array}{cc}
\operatorname{Re}\left[\mathbf{H}^{c}\right] & -\operatorname{Im}\left[\mathbf{H}^{c}\right] \\
\operatorname{Im}\left[\mathbf{H}^{c}\right] & \operatorname{Re}\left[\mathbf{H}^{c}\right]
\end{array}\right] \in \mathbb{R}^{2 N \times 2 M}, \mathbf{s} \triangleq\left[\begin{array}{c}
\operatorname{Re}\left[\mathbf{s}^{c}\right] \\
\operatorname{Im}\left[\mathbf{s}^{c}\right]
\end{array}\right] \in \mathbb{Z}^{2 M \times 1}, \\
& \mathbf{y} \triangleq\left[\begin{array}{c}
\operatorname{Re}\left[\mathbf{y}^{c}\right] \\
\operatorname{Im}\left[\mathbf{y}^{c}\right]
\end{array}\right] \in \mathbb{R}^{2 N \times 1}, \text { and } \mathbf{z} \triangleq\left[\begin{array}{c}
\operatorname{Re}\left[\mathbf{z}^{c}\right] \\
\operatorname{Im}\left[\mathbf{z}^{c}\right]
\end{array}\right] \in \mathbb{R}^{2 N \times 1}
\end{aligned}
$$

The set of the real-valued signals is given by $\mathbb{Z}=\{ \pm 1, \pm 3, \ldots, \pm(K-1)\}$ for $K^{2}$-QAM (Quadrature Amplitude Modulation). 


\subsection{LR-SIC detection}

In [3], Hassibi proposed an extend matrix form as

$$
\overline{\mathbf{y}} \triangleq\left[\begin{array}{c}
\mathbf{y} \\
\mathbf{0}_{2 M}
\end{array}\right], \overline{\mathbf{H}} \triangleq\left[\begin{array}{c}
\mathbf{H} \\
\sqrt{\gamma^{-1}} \mathbf{I}_{2 M}
\end{array}\right], \overline{\mathbf{z}} \triangleq\left[\begin{array}{c}
\mathbf{z} \\
-\sqrt{\gamma^{-1}} \mathbf{s}
\end{array}\right]
$$

where $\gamma=E_{s} / N_{0}$ with $E_{s}=E\left[\|\mathbf{s}\|^{2}\right] / 2 M$. $\mathbf{I}_{2 M}$ is a $2 M \times 2 M$ identity matrix and $\mathbf{0}_{2 M}$ denotes a $2 M \times 1$ vector with all zero entries. Then we have

$$
\overline{\mathbf{y}}=\overline{\mathbf{H}} \mathbf{s}+\overline{\mathbf{z}}
$$

The channel matrix $\overline{\mathbf{H}}$ can be QR-decomposed as $\overline{\mathbf{H}}=\mathbf{Q R}$ for the SIC operation. $\mathbf{Q}$ is a unitary matrix with $\mathbf{Q}^{\mathrm{T}} \mathbf{Q}=\mathbf{I}_{2 M}$ and $\mathbf{R}$ is a $2 M \times 2 M$ upper triangular matrix. Then the matrix $\mathbf{R}$ is transformed using the LLL algorithm in [6] as the LLL-reduced matrix $\tilde{\mathbf{R}}$ with $\tilde{\mathbf{Q}}$ and the transformation matrix $\mathbf{T}$. $\mathbf{T}$ is an integer unimodular matrix with $\operatorname{det}\{\mathbf{T}\}= \pm 1$. Due to a finite QAM constellation $\mathbb{Z}$ with odd lattice points, we assume that $\mathbf{s}$ is drawn from $\mathcal{A}^{2 M}$, of which $\mathcal{A}$ is a shifted and scaled set of the signals as $\mathcal{A}=\frac{1}{2} \mathbb{Z}+\frac{K-1}{2}$. First, scale $\overline{\mathbf{y}}$ as $\frac{\overline{\mathbf{y}}}{2}=\overline{\mathbf{H}} \frac{\mathrm{s}}{2}+\frac{\overline{\mathbf{z}}}{2}$. Then, shift the above as $\overline{\mathbf{y}}^{\mathcal{S}} \triangleq \overline{\mathbf{H}}\left(\frac{\mathrm{s}}{2}+\frac{K-1}{2} \mathbf{1}_{2 M}\right)+\frac{\overline{\mathrm{z}}}{2}$. The system model in (5) is rewritten as

$$
\begin{aligned}
\overline{\mathbf{y}}^{\mathcal{S}} & =\overline{\mathbf{H}}\left(\frac{\mathbf{s}}{2}+\frac{K-1}{2} \mathbf{1}_{2 M}\right)+\frac{\overline{\mathbf{z}}}{2}=\mathbf{Q R}\left(\frac{\mathbf{s}}{2}+\frac{K-1}{2} \mathbf{1}_{2 M}\right)+\frac{\overline{\mathbf{z}}}{2} \\
& =\tilde{\mathbf{Q}} \tilde{\mathbf{R}}\left(\mathbf{T}^{-1}\left(\frac{\mathbf{s}}{2}+\frac{K-1}{2} \mathbf{1}_{2 M}\right)\right)+\frac{\overline{\mathbf{z}}}{2} \equiv \tilde{\mathbf{Q}} \tilde{\mathbf{R}} \mathbf{v}+\frac{\overline{\mathbf{z}}}{2}
\end{aligned}
$$

where $\tilde{\mathbf{Q}} \tilde{\mathbf{R}}=\mathbf{Q R T}$ and $\mathbf{1}_{2 M}$ denotes a $2 M \times 1$ vector with all unit entries. The scaled and shifted vector of $\mathbf{s}$ is defined as $\mathbf{s}^{\mathcal{S}}=\mathcal{S}[\mathbf{s}] \triangleq \frac{\mathbf{s}}{2}+\frac{K-1}{2} \mathbf{1}_{2 M}$. Then we pre-multiple both the hand sides of (6) by $\tilde{\mathbf{Q}}^{\mathrm{T}}$ as

$$
\mathbf{y}^{\prime} \triangleq \tilde{\mathbf{Q}}^{\mathrm{T}} \overline{\mathbf{y}}^{\mathcal{S}}=\tilde{\mathbf{R}} \mathbf{v}+\mathbf{z}^{\prime}
$$

where $\mathbf{z}^{\prime} \triangleq \tilde{\mathbf{Q}}^{\mathrm{T}} \frac{\overline{\mathbf{z}}}{2}$. In the LR-SIC detection, the last entry of the new signal vector $v_{2 M}$ is first soft estimated as $\tilde{v}_{2 M}=y_{2 M}^{\prime} / \tilde{r}_{2 M, 2 M}$. Then it is quantized as the hard estimate $\hat{v}_{2 M}=\mathcal{Q}\left\{\tilde{v}_{2 M}\right\}$, where $\mathcal{Q}\{\cdot\}$ denotes the quantization operator. Assuming that the previous decisions are correct, the rest of the new signal vectors are derived in the following recursion as

$$
\hat{v}_{i}=\mathcal{Q}\left\{\tilde{v}_{i}\right\}=\mathcal{Q}\left\{\frac{y_{i}^{\prime}-\sum_{j=i+1}^{2 M} \tilde{r}_{i, j} \hat{v}_{j}}{\tilde{r}_{i, i}}\right\}, i=2 M-1, \ldots, 1
$$

The new signal vector $\hat{\mathbf{v}}$ is transformed back as $\hat{\mathbf{s}}^{\mathcal{S}}=\mathbf{T} \hat{\mathbf{v}}$. Then, $\hat{\mathbf{s}}^{\mathcal{S}}$ is shifted back and scaled back, expressing as $\hat{\mathbf{s}}:=\mathcal{S}^{-1}\left[\hat{\mathbf{s}}^{\mathcal{S}}\right]=2 \hat{\mathbf{s}}^{\mathcal{S}}-(K-1) \mathbf{1}_{2 M}$. The final decision $\hat{\mathbf{s}}$ is forced to the nearest constellation points if they are lying outside the original signal constellation as $\hat{\mathbf{s}}:=\mathcal{C}[\hat{\mathbf{s}}]$.

\section{Proposed OSIC with conditional list generation}

\subsection{Ordering the real lattice basis of channel matrix}

In [2], the optimal LR-OSIC has been proposed by updating the mean and variance of the estimated symbols $\tilde{\mathbf{v}}$ in the LR domain at each SIC stage. 
The estimate errors of the new signal vector in the LR domain correspond to the main diagonal entries of the error covariance matrix $\mathbf{\Phi}$, defined as

$$
\begin{aligned}
\mathbf{\Phi} & =E\left[(\tilde{\mathbf{v}}-\mathbf{v})(\tilde{\mathbf{v}}-\mathbf{v})^{\mathrm{T}}\right]=E\left[\tilde{\mathbf{R}}^{-1} \mathbf{z}^{\prime} \mathbf{z}^{\prime \mathrm{T}}\left(\tilde{\mathbf{R}}^{-1}\right)^{\mathrm{T}}\right] \\
& =\tilde{\mathbf{R}}^{-1}\left(\tilde{\mathbf{R}}^{-1}\right)^{\mathrm{T}} E\left[\mathbf{z}^{\prime} \mathbf{z}^{\prime \mathrm{T}}\right]=\frac{\sigma^{2}}{8}\left(\tilde{\mathbf{R}}^{\mathrm{T}} \tilde{\mathbf{R}}\right)^{-1}
\end{aligned}
$$

It is obvious that the smaller eigenvalue of $\tilde{\mathbf{R}}^{\mathrm{T}} \tilde{\mathbf{R}}$ will lead to more errors due to noise amplification. Let $\boldsymbol{\Psi}=\left(\tilde{\mathbf{R}}^{\mathrm{T}} \tilde{\mathbf{R}}\right)^{-1}$ in (9). Since $\tilde{\mathbf{R}}$ is the upper triangular matrix, we define $\tilde{\mathbf{R}}_{l}$ to be the partial square matrix denoting the partial columns (rows) of $\tilde{\mathbf{R}}$ from the first column (row) to the lth column (row), $l \in[2,2 M]$. In each stage. $\mathbf{\Psi}_{i}=\left(\tilde{\mathbf{R}}_{i}^{\mathrm{T}} \tilde{\mathbf{R}}_{i}\right)^{-1}$ is first obtained, where $i=2 M$ down to 2 . Then find out the minimum diagonal element of $\boldsymbol{\Psi}_{i}$. The specific signal vector having the lowest detection error variance is first detected. The column of $\tilde{\mathbf{R}}$ corresponding to the minimum diagonal element of $\boldsymbol{\Psi}_{i}$ should be swapped to the $i$ th column of $\tilde{\mathbf{R}}$ by turns. We apply the Givens rotation matrix $\Theta$ seen in [5] to keep the upper triangular matrix for $\tilde{\mathbf{R}}$. At the same time, $\tilde{\mathbf{Q}}$ is updated by multiplying $\boldsymbol{\Theta}^{\mathrm{T}}$. Until the iteration with $i=2$ is finished, finally output the updated $\tilde{\mathbf{Q}}$, updated $\tilde{\mathbf{R}}$ and $\mathbf{T}$.

Since the updated $\tilde{\mathbf{R}}$ has the information of detection error variance, the more reliable symbol is prior to being estimated in (8). At last, we need to transform the estimate signals to the original order as $\hat{\mathbf{s}}:=\mathcal{C}\left[\mathcal{S}^{-1}\left[\mathbf{T}^{-1} \hat{\mathbf{v}}\right]\right]$.

\subsection{Proposed scheme description}

The simplest quantization in (8) is the rounding operation. However the superior BER cannot be achieved only by rounding off the estimate of $\tilde{v}_{i}$. First, we observe the probability distribution of $\tilde{\mathbf{v}}-\hat{\mathbf{v}}$ including all the antennas' signals for the LR-OSIC in the $8 \times 8$ MIMO system.

As illustrated in Fig. 1, the probability distribution of $\tilde{\mathbf{v}}-\hat{\mathbf{v}}$ is close to the average distribution in the low $E_{b} / N_{0}$ region. On the contrary, the probability distribution of $\tilde{\mathbf{v}}-\hat{\mathbf{v}}$ approaches to the Gaussian distribution as $E_{b} / N_{0}$ increases. Hence we define the mean square error of the new signal vector in LR domain as $\varepsilon_{v} \triangleq \sum_{i=1}^{2 M}\left|\tilde{v}_{i}-\hat{v}_{i}\right|^{2}$. And define a threshold of the

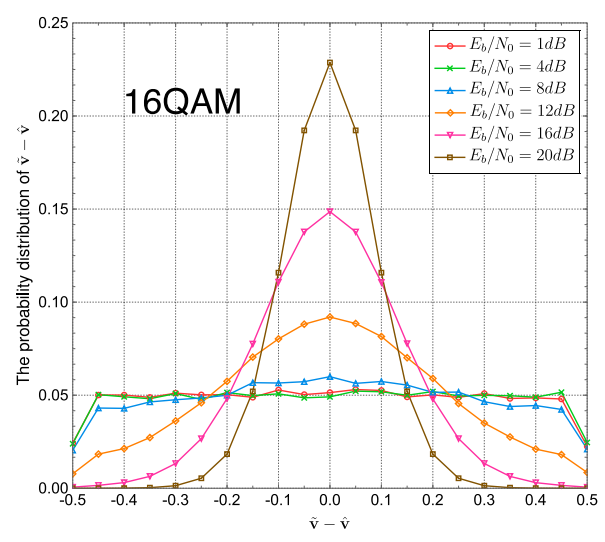

Fig. 1. Probability distribution of $\tilde{\mathbf{v}}-\hat{\mathbf{v}}$ in $8 \times 8$ MIMO. 


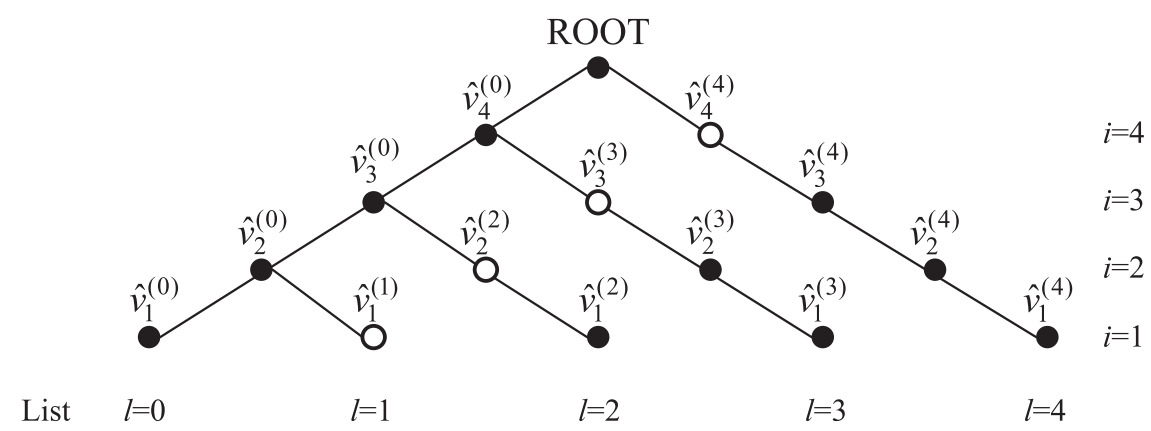

Fig. 2. The example of list tree in $2 \times 2$ MIMO.

mean square error $\varepsilon_{T H}$ as

$$
\varepsilon_{T H} \triangleq \Delta^{2} \cdot 2 M \text { with } \Delta^{2} \triangleq \frac{\sum_{i=1}^{2 M}\left|\tilde{v}_{i}-\hat{v}_{i}\right|^{2}}{2 M}
$$

where $\varepsilon_{T H}$ is predetermined and $\Delta^{2}$ denotes the average mean square error, of which the difference of $\left(\tilde{v}_{i}-\hat{v}_{i}\right)$ is $\pm \Delta$ to zero in Fig. 1. If $\varepsilon_{v} \leq \varepsilon_{T H}$, let the estimated symbols of the LR-OSIC be the final decision. Else we propose the conditional list detection to update the estimate of the LR-OSIC.

The list detection is proposed based on the soft estimate of the LROSIC. The list tree is generated in Fig. 2, where $l$ denotes the list number, $l \in[0,2 M]$. The estimated symbols in the list 0 are detected by the LROSIC, expressing as $\hat{\mathbf{v}}^{(0)}=\left[\hat{v}_{1}^{(0)}, \hat{v}_{2}^{(0)}, \ldots, \hat{v}_{2 M}^{(0)}\right]^{\mathrm{T}}$. The quantization error often happens in case that the difference of $\left(\tilde{v}_{i}-\hat{v}_{i}\right)$ is around $\pm 0.5, i \in[1,2 M]$. We give another candidate for each entry of the soft estimate of the LR-OSIC. Thus, the symbols $\hat{v}_{i}^{(l)}$ with $l=i$ marked white nodes in Fig. 2 are obtained by +1 or -1 on the rounding integer obtained by the estimate of LR-OSIC. The symbols in the $l$ th list $\hat{\mathbf{v}}^{(l)}=\left[\hat{v}_{1}^{(l)}, \hat{v}_{2}^{(l)}, \ldots, \hat{v}_{2 M}^{(l)}\right]^{\mathrm{T}}$ are defined as

$$
\hat{v}_{i}^{(l)}= \begin{cases}\hat{v}_{i}^{(0)} & l>i \\ \hat{v}_{i}^{(0)}+\operatorname{sgn}\left(\tilde{v}_{i}^{(0)}-\hat{v}_{i}^{(0)}\right) & l=i \\ \mathcal{Q}\left\{\tilde{v}_{i}^{(l)}\right\}=\mathcal{Q}\left\{\frac{y_{i}^{\prime}-\sum_{j=i+1}^{2 M} \tilde{r}_{i, j} \hat{v}_{j}^{(l)}}{\tilde{r}_{i, i}}\right\} & l<i\end{cases}
$$

Therefore the optimal estimate of new signal vector $\hat{\mathbf{v}}$ is chosen from $\hat{\mathbf{v}}=\left\{\hat{\mathbf{v}}^{(0)}, \hat{\mathbf{v}}^{(1)}, \ldots, \hat{\mathbf{v}}^{(2 M)}\right\}$, determined using the Euclidean distance (ED) as

$$
\begin{aligned}
\hat{\mathbf{v}}^{<\mathrm{opt}>} & =\underset{l \in[0,2 M]}{\arg \min } \sum_{i=1}^{2 M}\left|y_{i}^{\prime}-\tilde{r}_{i, i} \hat{v}_{i}^{(l)}-\sum_{j=i+1}^{2 M} \tilde{r}_{i, j} \hat{v}_{j}^{(l)}\right|^{2} \\
& =\underset{l \in[0,2 M]}{\arg \min } \sum_{i=1}^{2 M}\left|y_{i}^{\prime \prime(l)}-\tilde{r}_{i, i} \hat{v}_{i}^{(l)}\right|^{2}
\end{aligned}
$$

where the receive signal subtracted the interferences is defined as $y_{i}^{\prime \prime(l)} \triangleq$ $y_{i}^{\prime}-\sum_{j=i+1}^{2 M} \tilde{r}_{i, j} \hat{v}_{j}^{(l)}$.

Finally, we need to align back the estimate signals to the original order as $\hat{\mathbf{s}}:=\mathcal{C}\left[\mathcal{S}^{-1}\left[\mathbf{T}^{-1} \hat{\mathbf{v}}^{<\text {opt> }}\right]\right]$. Hence the ED calculated by the updated estimate of the new signal vector may be smaller than that calculated by the estimate of LR-OSIC, which results in the superior BER performance. 


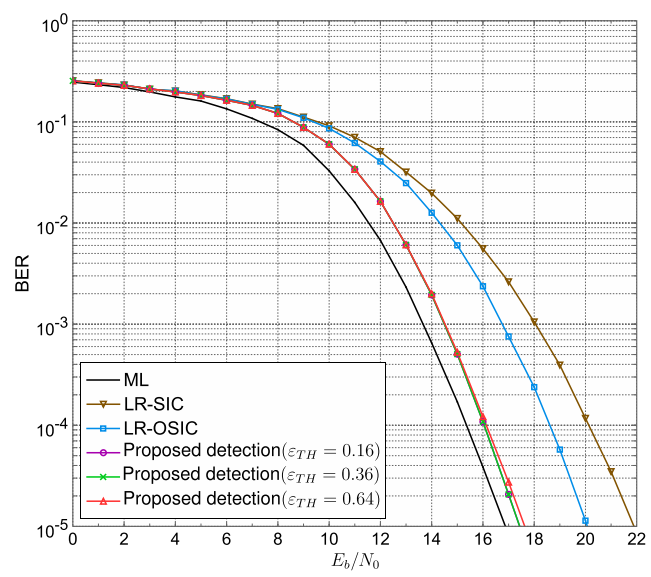

Fig. 3. The $E_{b} / N_{0}$ vs. BER characteristics for 16QAM.

\section{Numerical results}

We assumed that the channel is the typical flat Rayleigh fading. The computer simulations were carried out for $16 \mathrm{QAM}$ in the $8 \times 8$ MIMO system using the LR-SIC, the LR-OSIC, and the proposed detection with $\varepsilon_{T H}=$ $\{0.16,0.36,0.64\}$, respectively. In Fig. 3 , the curve of the proposed detection with $\varepsilon_{T H} \leq 0.36$ significantly improves $2.5 \mathrm{~dB}$ gain compared with that of the LR-OSIC at the BER of $10^{-5}$, which approaches the curve of ML detection.

Without the ordering operations, the LR-SIC and the LR-OSIC have the same complexity in the signal estimation stage. The complexity for computing $\mathbf{y}^{\prime}$ in (7) and $\hat{\mathbf{v}}$ in (8) is counted up as $\left(8 M^{2}+8 M N-2 M\right)$ and $4 M^{2}$ flops, respectively. Hence, the total complexity of the LR-OSIC including the scaling and shifting operations is $\left(20 M^{2}+8 M N+4 M+4 N\right)$ flops, which requires 1856 flops for $M=N=8$. The complexity for the list generation is $2 M$ and $\left(\frac{16 M^{3}-M}{3}-2 M^{2}\right)$ flops for computing the candidates with $l=i$ and the rest symbols with $l<i$ in (11), respectively. And the optimal decision in (12) requires $8 M$ flops for computing the path metric of the LR-OSIC and $\left(8 M^{2}+4 M\right)$ flops for that of the list candidates. The total extra complexity of the conditional list generation requires $\left(\frac{16 M^{3}+41 M}{3}+6 M^{2}\right)$ flops.

We observed the percentage of $\varepsilon_{v} \leq \varepsilon_{T H}=0.36$ for the proposed detection, which implied the percentage of the estimate of LR-OSIC to be the final decision without the list generation. The percentages of $\varepsilon_{v} \leq \varepsilon_{T H}$ were about $88 \%$ corresponding to the BER of $10^{-4}$, and about $96 \%$ corresponding to the BER of $10^{-5}$, respectively. Therefore, the conditional list detection required very lower complexity at the BER below $10^{-4}$.

\section{Conclusion}

In this paper, we proposed a low complexity MIMO detection using the OSIC with conditional list generation. The list tree was generated to update the soft estimate of the LR-OSIC in the condition of $\varepsilon_{v}>\varepsilon_{T H}$. The simulation results exhibited that the proposed detection achieved the near-ML BER performance with almost the same complexity of the LR-OSIC in the high $E_{b} / N_{0}$ region in the $8 \times 8$ MIMO system. 